\title{
Bilateral chylothorax in an AIDS patient with newly diagnosed Kaposi sarcoma
}

\author{
Rebecca E Neril, MD, and Kimberly Lam, MD
}

Department of Internal Medicine, SBH Health System, Bronx, New York

K aposi sarcoma is an angioproliferative tumor that is associated with human herpes virus-8 (HHV-8). Mucocutaneous disease is the most common site for manifestation of AIDSrelated Kaposi sarcoma, commonly affecting the lower extremities, oral mucosa, face, and genitalia. Pleural effusions can occur in $36 \%-60 \%$ of patients with Kaposi sarcoma, and it has been documented that chylothorax is a rare, but plausible presentation in patients with Kaposi sarcoma. ${ }^{1}$ We present here a case of bilateral chylothorax in a patient with AIDS-related Kaposi sarcoma.

\section{Case presentation and summary}

A 52-year-old MSM male with AIDS (CD4, <20 $\mathrm{mm}^{3}$; viral load, 58 copies $/ \mathrm{ml}$ ) presented to the emergency department with complaints of shortness of breath, productive cough, and diarrhea for 2 days prior to presentation. His medical history also included chronic obstructive pulmonary disease, coronary artery disease, and hyperlipidemia. The patient was not on HAART because of his history of noncompliance. The results of a chest $\mathrm{X}$-ray and computed-tomography (CT) scan showed that the patient had bilateral pleural effusion and a spiculated 14-mm nodule in the left upper lobe. The patient underwent ultrasound-guided placement of a 12-French left-sided chest catheter, and a milky white fluid was aspirated from the left pleural space. Laboratory analysis of the pleural fluid confirmed an exudate with an elevated triglyceride level of $120 \mathrm{mg} / \mathrm{dL}$ (chylous, >110 mg/dL) indicating chylothorax.

On close physical examination, the patient was found to have multiple irregular plaques on the back and lower extremities. As described by dermatology, there was a violaceous indurated plaque on the left axillae, violaceous indurated plaques with superficial scale grouped on the left midlateral back, and hyperpigmented lichenified plaques and papules on bilateral shins, with some with plate-like scale. Two punch biopsies were taken of the skin lesions, which confirmed Kaposi sarcoma, plaque stage from the lesion biopsied on the back, and patch stage from the lesion biopsied in the left axilla. Cytology of the pleural fluid was negative for malignant cells. On review by the radiologist of the $\mathrm{CT}$ scan of the chest, there was no indication of gross distention of the thoracic duct. Treatment options were offered to the patient, and the patient was considering options for chemotherapy and home hospice given his advanced disease state at the time of discharge.

\section{Discussion}

Chylothorax occurs with a thoracic duct obstruction, which results in leakage of lymphatic fluid into the pleural cavity. The two leading causes of chylothorax are trauma and malignancy, with lymphoma being the most common cause of chylothorax among those with malignancy. ${ }^{2}$ Chylothorax, however, is a rare but documented complication of Kaposi sarcoma. Marais and colleagues reported the case of a 3-year-old HIV-positive patient with newly diagnosed Kaposi sarcoma who was found to have tumor infiltration in the thoracic duct leading to bilateral chylothorax. ${ }^{3}$ Maradona and colleagues described a 40-year-old man with AIDS-related Kaposi sarcoma who was found to have pleural and pericardial Kaposi sarcoma with chylothorax. ${ }^{4}$ Priest and colleagues wrote about a 32-yearold patient with AIDS with biopsy-proven Kaposi sarcoma who required multiple therapeutic thoracenteses for rapidly recurrent left chylothorax effusions. ${ }^{5}$

There are two leading discussions as to the pathophysiology of chylothorax that is related to Kaposi sarcoma: chylothorax developing secondary to metastatic disease or the development of chylotho-

Accepted for publication April 11, 2016. Correspondence: Rebecca E Neril, MD; Rebecca.neril@gmail.com. Disclosures: The authors report no disclosures or conflicts of interest. JCSO 2017;15(3):e174-e175. (02017 Frontline Medical Communications. doi: https://doi.org/10.12788/jicso.0261 


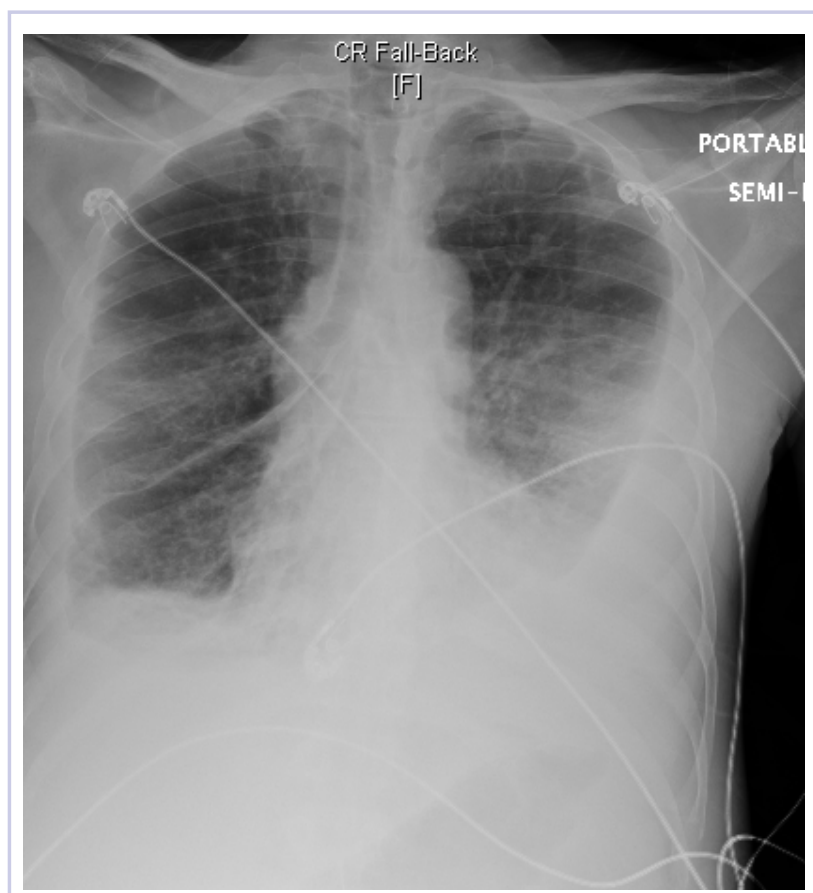

FIGURE 1 Chest X-ray showing bilateral pleural effusion blunting the costophrenic angles.

rax secondary to primary Kaposi sarcoma arising from the pleural region. ${ }^{6}$ One case report examined pleural and lung biopsies in a 34-year-old patient with AIDSrelated Kaposi sarcoma that showed immunohistochemical staining that was suggestive of early-stage Kaposi sarcoma of lymphatic endothelial origin. The authors were

\section{References}

1. Sridar S, Garza EG, Cox J, Rumbak MJ. Serosanguineous pleural effusions in a patient with HIV and Kaposi sarcoma: pleuroscopic findings. J Bronchology Interv Pulmonol. 2011;18(4):337-339.

2. Light RW. Chylothorax and pseudochylothorax. In: Light RW, ed. Pleural diseases. 6th ed. Philadelphia: Lippincott Williams \& Wilkins, 2013:412-426.

3. Marais BJ, Pienaar J, Gie RP. Kaposi sarcoma with upper airway obstruction and bilateral chylothoraces. Pediatr Infect Dis J. 2003;22:926-928.

4. Maradona JA, Carton JA, Asensi V, Rodriguez-Guardado A. AIDS-

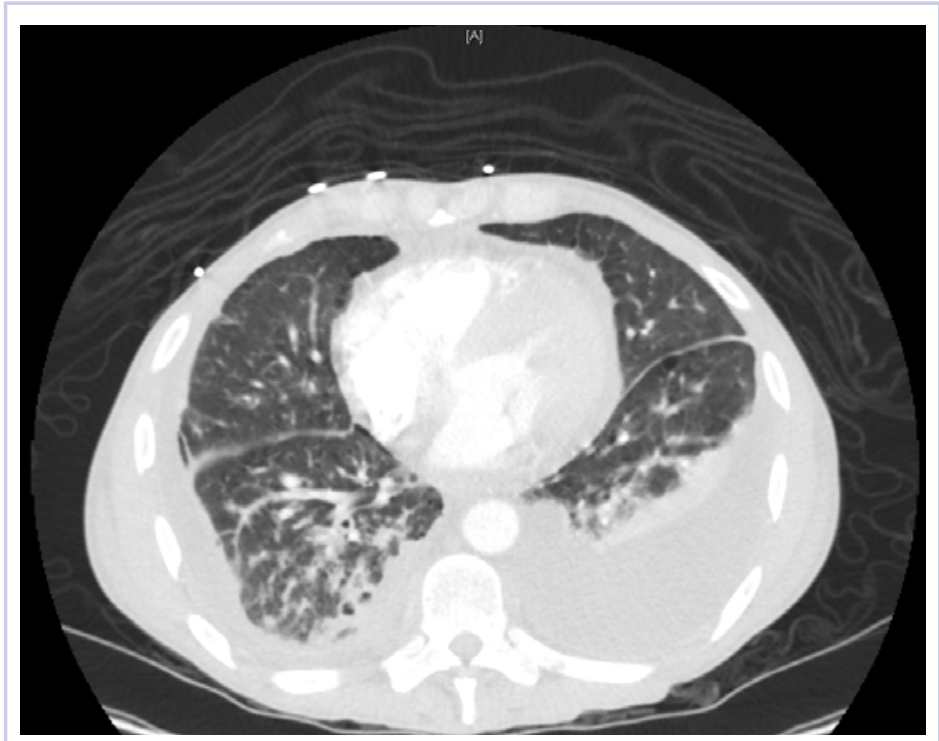

FIGURE 2 A helical computed-tomography scan of the chest showed bilateral pleural effusion.

attempting to illustrate that Kaposi sarcoma may have a stem-cell origin which can differentiate into lymph cells. Kontantinopoulos and colleagues postulated that in situ Kaposi sarcoma can arise from the lymphatic system with a resultant clinical presentation of chylothorax. ${ }^{7}$ The more mainstream thought however, is that chylothorax has been found to develop secondary to metastatic disease. The present case, therefore, illustrates an unusual presentation of cytology negative chylothorax in a patient with AIDSrelated Kaposi sarcoma.

related Kaposi sarcoma with chylothorax and pericardial involvement satisfactorily treated with liposomal doxorubicin. AIDS. 2002;16(5):806.

5. Priest ER, Weiss R. Chylothorax with Kaposi sarcoma. South Med J. 1991;84:806-807.

6. Pantanowitz L, Dezube BJ. Kaposi sarcoma in unusual locations. BMC Cancer. 2008;8:190.

7. Konstantinopoulos PA, Dezube BJ, Pantanowitz L. Morphologic and immunophenotypic evidence of in situ Kaposi sarcoma. BMC Clin Pathol. 2006;30:6:7. 